\title{
A new method for the acquisition of arterial pulse wave using self-mixing interferometry
}

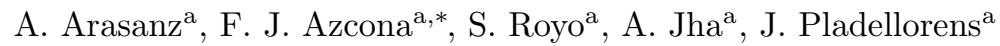 \\ ${ }^{a}$ Centre for Sensors, Instruments and Systems Development (CD6),UPC-BarcelonaTech, \\ Rambla St. Nebridi 10, 08222, \\ Terrassa, Spain.
}

\begin{abstract}
In this article we present a technique based on self-mixing interferometry as a method for the acquisition and reconstruction of the arterial pulse wave. A modification, of the classic fringe counting reconstruction algorithm is proposed, to deal with some of the problems caused by biological tissue surface roughness, therefore allowing a reconstruction of the arterial displacement with a resolution of 400nm. The traits of the arterial pulse wave have been retrieved with high detail, allowing their interpretation by a skilled practitioner. The heart beat measurements show a good agreement when compared to the readings of a commercial pulse-meter, therefore proving the versatility and the viability of the technique for the measurement of other cardiovascular signals.
\end{abstract}

Keywords: arterial pulse wave, cardiovascular measurement, laser sensors, self-mixing interferometry, signal processing

\section{Introduction}

In recent years, different non-invasive and non-contact methods for the acquisition of biomedical signals have been developed in order to increase the quality of patient care [1-3]. Techniques based on optic sensors are an interesting solution for biomedical signal measuring because of their simple construction, resolution, bandwidth, and low cost. Optical methods, in particular, have been assessed as a suitable solution for the measurement of different cardiovascular signals such as the cardiac rhythm and the arterial pulse wave $[4,5]$.

The development of non-invasive methods for the acquisition and interpretation of cardiovascular signals has gained importance in the last years because of the increase in cardiovascular diseases around the world. As it is described in [5], the arterial pulse wave is one of the most important signals used on clinical medicine. The analysis of this signal can lead to the diagnosis of several cardiovascular diseases such as arterial stiffness $[6,7]$ and arteriosclerosis [8]. Different optical methods have been previously

\footnotetext{
This document is a collaborative effort.

* Corresponding author.

Email addresses: a.arasanz@alu-etsetb.edu (A. Arasanz), francisco.javier.azcona@cd6.upc.edu (F. J. Azcona ), santiago.royo@upc.edu (S. Royo), ajit.jha@cd6.upc.edu (A. Jha), pladellores@oo.upc.edu (J. Pladellorens)

${ }^{1}$ Phone: +34 937398905
} 
proposed for the acquisition of the arterial pulse wave, being the photoplethysmography [9-11] and the Doppler based methods $[4,12,13]$, the most common solutions for the task.

Self-mixing interferometry $(S M I)$, also known as optical feedback interferometry $(O F I)$, has been previously assessed as a non-invasive technique for the pulse detection based on laser Doppler vibrometry $(L D V)[4,14,15]$. In this article, however, we use a more direct interpretation of the SMI signal focusing on the amplitude instead of the frequency, therefore allowing a complete reconstruction of the arterial pulse shape. Thus, in this work, we consider the cardiovascular pulse as a pressure wave which induces changes in the radius of the arterial wall in a similar way as the one proposed by Hast[4]. Such changes may induce a small displacement in the order of a few microns over the skin surface which can be captured by an SMI interferometer. As it will be shown, the reconstructed waveforms show similar resolution as the one presented by photoplethysmography $(P P G)$ methods.

In the following section, we introduce the elements required for the proposed method as well as a short summary of the SMI method and how it can be applied to the reconstruction of the arterial pulse wave. In Sec.3, we discuss some of the main aspects regarding the processing algorithm for the SMI, as well as some precautions that should be taken to ensure an efficient readout of the pulse waveform. In Sec.4, we show a summary of the results attained while using the SMI method. Finally we establish the current state of the proposed method and the intended future work.

\section{Material and methods}

\subsection{Self-mixing interferometry}

Self-mixing interferometry has been an active area of research since the early 1980s. The phenomenon was first characterized by Lang and Kobayashi [16], who analysed the effects of external optical feedback in a laser diode (LD) by means of the delayed differential equations of involved electric field. Later models, such as the one presented by Wang [17] (Fig.1), use an equivalent two cavity Fabry-Perot (FP) scheme that also results in the well known phase equation [18]:

$$
2 \pi \tau_{\text {ext }}\left(\nu-\nu_{0}\right)=C \sin \left(2 \pi \nu \tau_{\text {ext }}+\arctan \alpha\right),
$$

where the effects of the light round trip time $\tau_{\text {ext }}$, the emission frequency of the free-running laser $\nu_{0}$, the emission frequency after feedback $\nu$, the feedback factor $C$ and the linewidth enhancement factor $\alpha$ over the SMI signal can be analysed.

In practice, the SMI can be described as the modulation of the laser optical output power (OOP) given by:

$$
P=P_{0}\left(1+m \cos \left(2 \pi \nu \tau_{e x t}\right)\right),
$$

where $P$ is the OOP, $P_{0}$ the initial output power and $m$ a modulation coefficient, produced when part of the light backscattered by a target re-enters the laser cavity. Depending on the amount of feedback [19], 


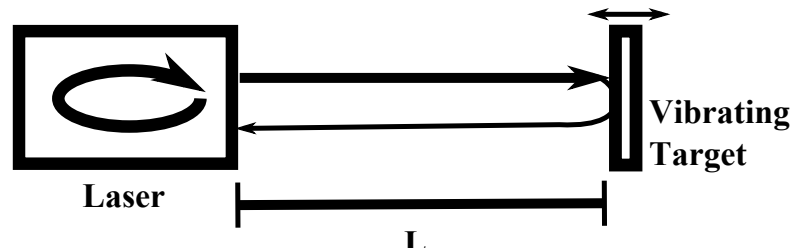

Figure 1: A schematic of the three mirror configuration of SMI. The thin line represents the backscattered light that re-enters the laser cavity

which is accounted by $C$ and mathematically described in Eq.(3), where $\tau_{l}$ represents the round trip time inside the laser cavity, $R_{e x t}$ and $R_{s}$ the reflectivity of the target and of the emitting facet respectively and $\epsilon$ the fraction of light producing the interference; the form of the modulation can change.

$$
C=\epsilon \frac{\tau_{e x t}}{\tau_{l}} \frac{\sqrt{R_{e x t}}}{R_{s}}\left(1-R_{s}\right) \sqrt{1+\alpha^{2}} .
$$

For values of $C<0.1$ (very weak regime) Fig.2B the signal has a purely sinusoidal shape. For $0.1<C<1$ (weak regime) shown in Fig.2C, the sinusoidal shape is distorted and it, progressively, acquires a saw-tooth like shape as $C$ increases. For $C>1$ (moderate regime) shown in Fig.2E the SMI signal behaves as a saw-tooth and as the $C$ value increases, it can suffer from hysteresis and fringe loss [20]. Finally at high feedback levels Fig.2F, the OOP enters into chaotic regime, where it is no longer possible to extract information related to the target displacement. For most SMI applications, it is preferred to work close to the boundary between the weak and moderate regimes Fig.2D, which results in a good signal to noise ratio $S N R$ and enables an easier detection of the transitions in the signal. In this kind of detection, each transition is equivalent to a half-wavelength $(\lambda / 2)$ displacement [18], and the slope preceding the transition enables to infer the direction of the displacement.

Although the SMI can be produced using different laser types, the most common SMI setups rely on the use of semiconductor lasers, typically preferring single mode lasers that include a monitor photodiode (PD) in the same package as the LD. Thus, the LD acts simultaneously as source and detector, avoiding the need for complex alignment procedures. The use of this arrangement makes the system extremely robust and compact. Two measurement schemes can be applied for the recovery of the SMI signal [21]; nevertheless, the so-called PD scheme, applied in this work, is preferred because of its higher sensitivity when compared to the scheme based on junction voltage detection.

\subsection{Materials}

The measurements described on the following sections were carried out using a Hitachi FP LD GaAlAs HL7851G with nominal wavelength of $785 \mathrm{~nm}$ and output power of $50 \mathrm{~mW}$ placed as shown in Fig.3. The LD is placed on a laser tube that contains a thorlabs aspheric C230220P-B lens and it is connected to a continuous current driver. The SMI signal is recovered using the laser internal PD which is connected to a transimpedance amplifier. After a preconditioning and an electronic gain stage, the signal is acquired using a Tektronix DPO2024 oscilloscope. All the signals were analysed using a custom software created 

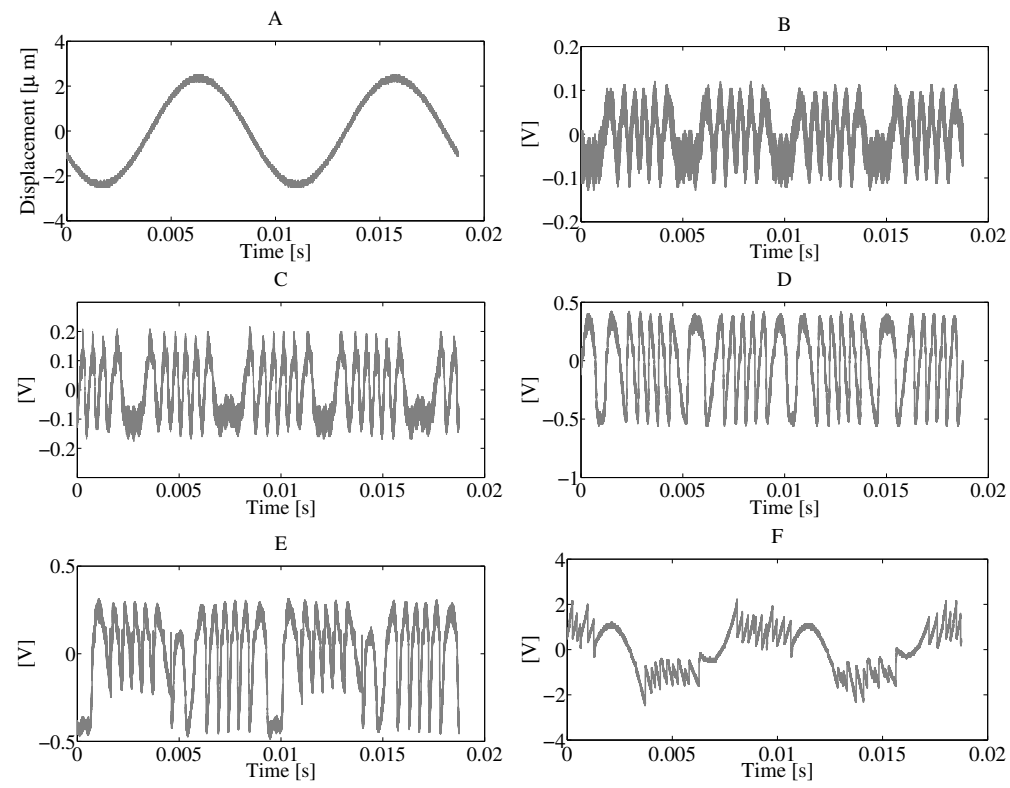

Figure 2: Self mixing signals for different regimes of a given sinusoidal target displacement represented in A. B very weak regime, $\mathrm{C}$ weak regime, $\mathrm{D}$ close to the boundary between weak and moderate regime $(C \approx 1), \mathrm{E}$ moderate regime and $\mathrm{F}$ strong regime.

on MATLAB. Ex-vivo measurements were obtained using a PI-LISA piezoelectric stage $(P Z T)$ controlled a E-816 driver. For the heart beat frequency measurements, a commercial sport pulse-meter FT4 Polar with a refreshing rate of 30s was also used for comparison purposes.

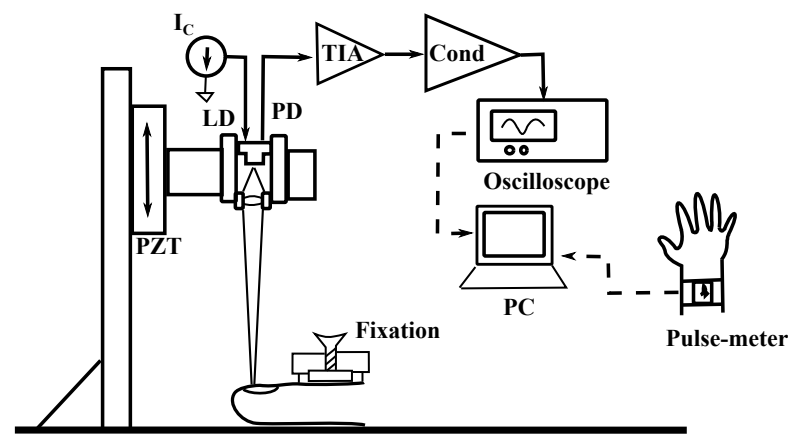

Figure 3: Schematic setup. The broken lines to the PC represent that the collected measurements are registered using an external usb memory device (oscilloscope measurements), or manually after a visual reading (pulse-meter). Connections to the PZT driver are not represented.

During all the experiments, the LD is mounted on a holder and mechanically attached to the PZT in order to perform measurements on the vertical axis as shown on Fig.3. For in-vivo measurements, the measured surface was fixed by a small aluminium piece to reduce possible artifacts caused by involuntary motion. When the part to be measured is in place, the SMI signal is calibrated to a feedback regime close to $C=1$ by a visual inspection. The calibration consists on an adjustment of the laser spot size, 
which can be achieved either producing a small change on the focusing lens, or by a change of the PZT position.

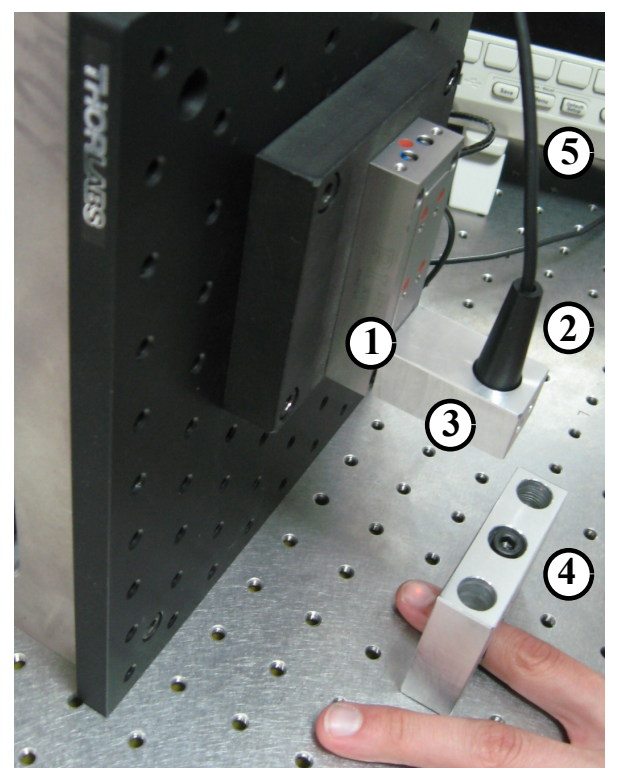

Figure 4: Experimental setup for the measurement of arterial pulse wave. 1. PZT; 2. Laser tube with lens and LD, the LD is wired to an electronic card; 3. LD mechanical holder; 4. Finger mechanical fixation; 5 . Oscilloscope.

It is possible to automate the calibration process by applying one of the methods proposed by Atashkhooei [22]. It is also important to remark that several more user friendly approaches may be applied for finger immobilization such as the finger clip used in available photoplethysmography methods. To reduce the effects of involuntary tremors over the measurement, it is also possible to apply a high-pass filter with a cut-off frequency around $0.5 \mathrm{~Hz}[23]$. All the measurements were performed placing the LD at approximately $10 \mathrm{~cm}$ from the target. The technique can be applied at a longer distance if a collimation lens is used instead of a focusing lens.

For the heart beat measurements, the test subject was asked to jog during a couple of minutes before the test to increase his heartbeat. The measurements were produced every thirty seconds to match the sampling rate of the pulse-meter. A comparison between both measurements is presented in a following section.

\section{Calculation}

As it was described in the introduction, in this study we apply the displacement approach of the SMI method rather than the Doppler approach commonly used on biomedical studies. The use of this type of measurement allows the retrieval of the complete arterial pulse wave contour in an almost direct fashion, thus, not only limiting the application to the detection of the velocity in the blood flow.

The most common approach for the reconstruction of a signal in the SMI displacement method is the so-called fringe counting algorithm (FCA). The FCA typically recovers the signals in three steps Fig.5: 
first the transitions on the SMI signal are detected (typically applying a derivative process and comparing the result with a threshold value), then, a discrete step of $\lambda / 2$ is set at every transition according to the positive or negative value of the transition and finally a smoothing process may be applied over the reconstructed signal. If higher resolution is required, other types of reconstruction processes may be applied such as the ones discussed in [24, 25].
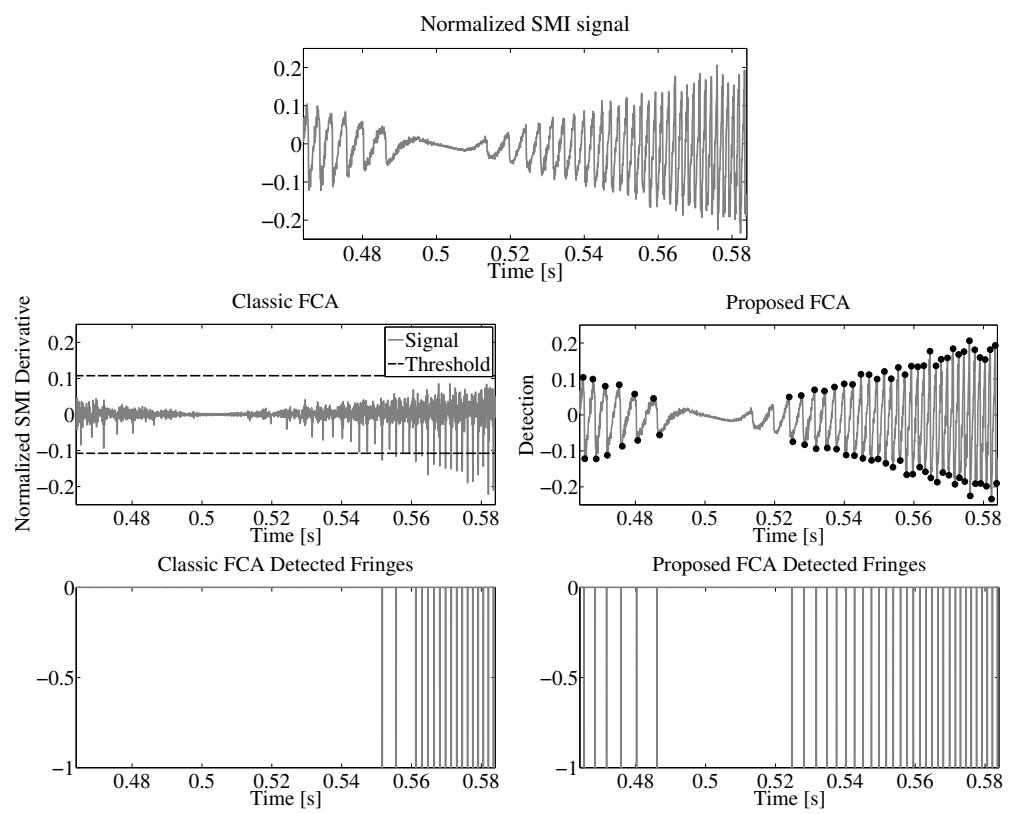

Figure 5: Example of the fringe counting algorithm applied to a SMI signal. On the left, the signal fails recovering all the fringes because of the speckle. On the right, the new algorithm recovers most of the transitions of the same signal.

The FCA is highly efficient in the measurement of homogeneous surfaces. Nevertheless, in the case of heterogeneous surfaces, particularly those with high surface granularity (such as the skin and other biological tissue), the FCA can produce faulty results. These results are generated because of the appearance of speckle [26] over the SMI signal, thus, not allowing the use of a continuous threshold level throughout the SMI signal and increasing the complexity and resource consumption of the reconstruction algorithm. This may result, in some cases, in the loss of displacement information. Some methods have already been proposed in order to reduce the influence of speckle [27, 28]; however, in both cases, the setups require the use of external elements (e.g. other LDs or tracking elements), thus, increasing the complexity of the sensor. For the work presented in this article we propose the use of a simple modification of the FCA algorithm in order to limit the effects of speckle over the SMI signal.

The proposed FCA changes the detection stage described before as follows. In a first step, the SMI signal is pre-processed using a low pass-filter with cut off frequency in the order of $1 \mathrm{kHz}$ to reduce high frequency noise effects, then, the local maxima and minima of the SMI signal are detected, as shown with the black dots on Fig.5, and their coordinates are stored in a vector .

Once the maxima and minima of the signal are detected, the algorithm computes the slopes $\left(m_{1}\right.$ and 
$m_{2}$ ) of three consecutive maxima and minima points as:

$$
m_{1}=\left|\frac{y_{2}-y 1}{x_{2}-x 1}\right| \text { and } m_{2}=\left|\frac{y_{3}-y_{2}}{x_{3}-x_{2}}\right|,
$$

where $y_{k}$ is the value of the point, and $x_{k}$ its time of appearance. Then, $m_{1}$ and $m_{2}$ are compared. The largest value $m_{k}$ is selected as the place where the transition occurs. A positive or negative sign is assigned to each transition by comparing the values of the points defining the slope $m_{k}$. In the case $y_{k}<y_{k+1}$ the transition is considered as positive. The transition is then stored in a vector on the position $x_{k}+\left(x_{k+1}-x_{k}\right) / 2$. Finally, the index of the coordinate vector is increased in two and the process is repeated for all the detected maxima and minima.

After the fringe detection, FCA reconstructs the arterial pulse profile by numerical integration considering that each transition senses a $\lambda / 2$ displacement. Finally, a smoothing process using a low pass filter may be applied to refine the reconstructed waveform.

\section{Results}

In order to test possible errors in the arterial pulse wave reconstruction procedure or in the proposed setup, an artificial pulse is generated using a piezoelectric stage with a displacement that follows the behavior of a typical arterial pulse. The maximum amplitude applied for the artificial pulse is $19 \mu m$ and $1 \mathrm{~Hz}$ frequency to keep the values as close as possible to those present in human pulse. On top of Fig.6, the superposition between the artificial pulse and the reconstructed signals can be appreciated, and, on the bottom, the error between the generated and the reconstructed signals is shown. It is remarkable to notice that the larger errors correspond to points where there is a large change in slope due to the OOP humps which appear when the direction of the target movement changes.

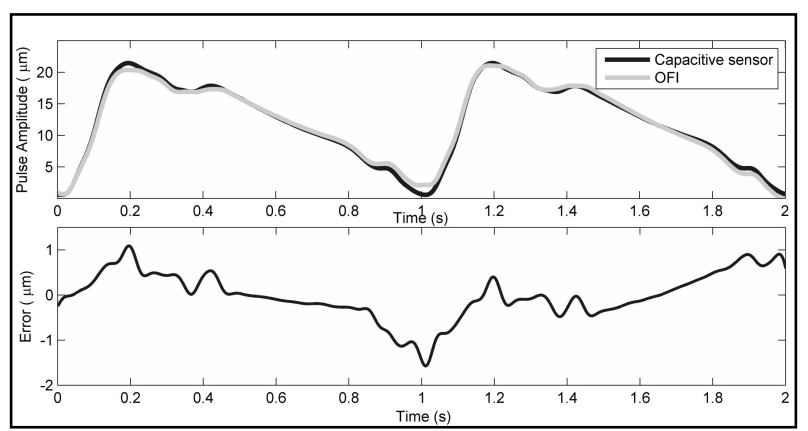

Figure 6: Difference between artificial pulse and SMI reconstruction

Another factor to take into account for correct arterial pulse wave reconstruction with the SMI method is the use of a proper sampling rate. To test the effects of sampling frequency on signal reconstruction, a set of different sampling rates was analyzed. As shown on Fig.7, where an intermediate $(31.25 \mathrm{kS} / \mathrm{s})$, and the lowest sampling frequency studied $(3.125 \mathrm{kS} / \mathrm{s})$ are shown, no relevant differences in the reconstructed waveforms are appreciated, except for some small high-frequency details at the top of the arterial pulse. As depicted, absolute errors in amplitude are lower than $2 \mu \mathrm{m}$ in both cases and the shape of the pulse 
is properly reconstructed for most of the points in the acquired signal. Taking a closer look into the artificial pulse, it is possible to estimate that the minimum sampling rate to hold Nyquist criteria is close to $1 \mathrm{kS} / \mathrm{s}$ for the rise on the arterial pulse wave signal.
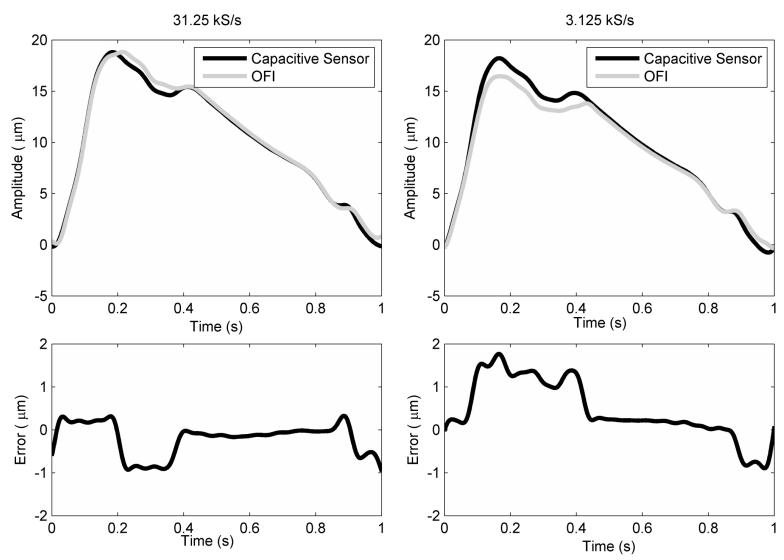

Figure 7: Effect of the sampling frequency over the SMI reconstruction.

\subsection{In-vivo arterial pulse wave measurement}

The measurements were performed following the procedure depicted in Sec.2 and following the sampling directives established on this section. The laser was pointed at the index fingernail of the left hand of the first author of this paper. The data is then processed using the algorithm discussed on Sec.2.2. An example of the typical reconstructed signal and the result of the fringe detection algorithm are shown on Fig.8.

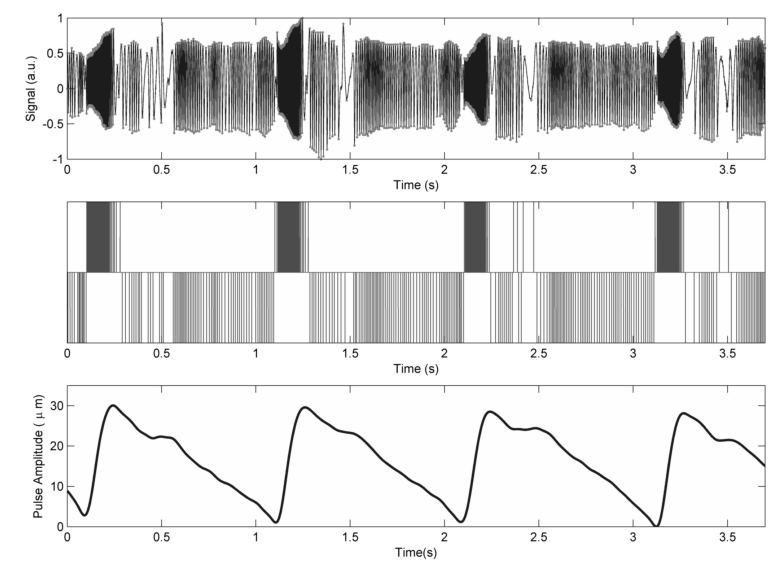

Figure 8: Steps for the reconstruction of the arterial pulse wave. From top to bottom: the SMI pre-filtered signal, the fringe detection and the recovered arterial pulse.

It is important to remark that, even when a small amount of speckle may be observed in the SMI signal, it was still possible to perform a correct detection of all the interferometric fringes. In the top of Fig.8, the measured SMI signal is shown. As it is expected because of the higher velocity of the displacement during the ascent line (e.g. $\mathrm{t}=0.1$ to $\mathrm{t}=0.2 \mathrm{~s}$ ), a higher density of the SMI signal and the 
detected fringes can be observed. This marks the beginning of the arterial pulse wave. Once the arterial pulse wave reaches its maxima, it is possible to observe a decrease on the velocity (shown by lower density fringe zones), which correspond to the line of descent, represented by the negative SMI fringes. It can also be noticed that during the descent, the dicrotic notch is clearly shown in all cases either by a reduction of the speed (zones with the lowest density of fringes) or by a change of slope (represented by the appearance of positive fringes during the line of descent).

If the measurement is performed with $C \approx 1$ with the finger at rest, better results can be expected, reducing the possibility of a detection failure caused by artifacts. A complete reconstruction of the arterial pulse wave is displayed in the bottom of Fig.8. As shown, the reconstructed pulses are well characterized presenting a fast ascent, decreasing to the dicrotic notch and finally descending to the base of the next pulse. The obtained waveforms are comparable to the signals typically obtained on PPG sensors.

\subsection{Pulse frequency measurement}

By implementing the Fast Fourier Transform (FFT) over the reconstructed arterial pulse, the main frequency component of the pulse can be retrieved with high accuracy. This method allows us to compare our results, at least to some extent with those obtained by commercially available detectors.

In order to prove that the estimated beat of the obtained signal corresponds to the heart beat rate, a comparison with a heartbeat rate monitor (a commercial sport pulse-meter) was performed. In Fig.9, a comparison between the measurement results of both devices is depicted. As it may be observed by the linear fit, both measurements show good agreement. Differences on the measurement may be explained by the length of the signal used on the FFT, as it averages the effects caused by single time events. It is also important to note that the refresh rate of the proposed sensor is higher than the one of the pulse-meter, which might also explain the differences between measurements.

\section{Discussion}

As presented in the introductory section, there are several methods capable of measuring the arterial pulse wave. The results obtained with the proposed method are similar to those obtained with PPG methods but using SMI, therefore allowing, in theory a non-contact approach.

Taking a look at the SMI literature on the topic, Hast $[4,13]$ has already documented the possibility of using a SMI sensor in a LDV configuration for cardiovascular measurements. Their work has shown that there is a high degree of correlation between the derivative of the pressure pulse wave and the Doppler spectrogram of the arterial blood velocity. Their measurements were performed over the wrist and the sensor was aligned using a loud speaker. In our opinion, this is one of the main differences between their work, and our results. In this work we propose the measurement over a fingernail instead of the wrist. While the latter seems as a more suitable location for the measurement of cardiovascular signals, it is also clear that it is harder to predict the behaviour of the SMI signal over the skin because 


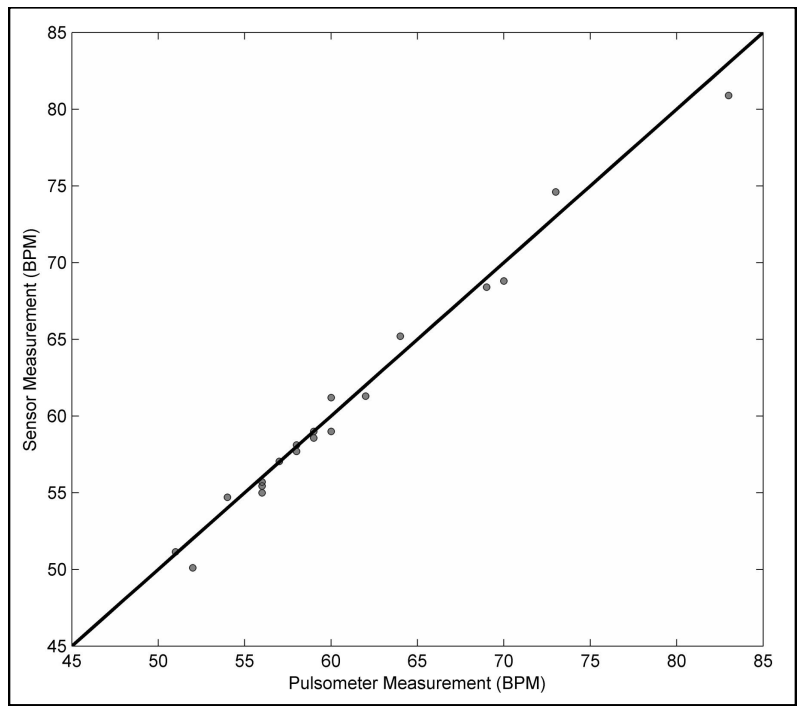

Figure 9: Comparison between heart beat measurement obtained by a commercial pulse-meter and the proposed SMI method (represented in dots). The solid line shows a linear fit of the measurements showing a good agreement between both sensors. The heart beat measurements are performed in parallel to the arterial wave pulse measurements.

of its roughness and pigmentation, therefore increasing the adaptability requirements of the measuring instrument. By measuring over the fingernail, in our opinion, the adaptability issues are reduced due to nail homogeneity and better reflectivity when compared to skin tissue.

Another important difference between the LDV and the proposed measurement relies on the placement of the interferometer. While measurements proposed by Hast are performed almost directly over the skin and with an angle between the interferometer and the skin surface, we have performed the measurements at a $10 \mathrm{~cm}$ distance normal to the target surface. This shows that in principle it may be possible to produce a non-contact measurement; nevertheless, in practice this is difficult to achieve since all motions larger than $\lambda / 2$ are captured by the sensor. In practice a mechanical fixation such as the one proposed in the article may reduce this effects.

In comparison to PPG measurements, we think the main difference relies on the number of optical elements needed on each setup. While PPG sensors require an external photodiode to capture the signal, SMI relies solely on a LD. While the measurements presented in this article were performed using the PD inside of the laser, this element is not necessary to perform the measurement as it is noted in [21]. It is also to be noted, that not only the electronic measurement scheme can be changed, but also the laser used for the measurements. The use of VCSEL lasers for this type of application may be interesting due to their lower output power and consumption. This, however, may reduce the SNR requiring further processing of the SMI signal. From the experience of this work, it might be possible to produce measurements with SNR values around $3 d B$ using the proposed algorithm.

From the results presented in Sec.4, the higher discrepancies between the reconstruction and the arterial pulse are coincident with the lobes (local maxima and local minima) of the arterial pulse wave. On this points, the phase changes produced by the pulse wave are lower than the $\lambda / 2$ resolution of SMI. 
This limitation has to be further studied; however, the use of other processing methods, such as the phase unwrapping algorithm, might reduce the effects of the error over the measurement. Heartbeat frequency measurements were also used for comparison purposes. As it is observed, the attained measurements show a good agreement with those measurements obtained by a commercial pulse-meter.

Further work is required in order to built a complete sensor. A better mechanical model still has to be studied to have a better understanding of the interactions between the nail and the underlying skin tissue. In this study we considered the fingernail as a rigid structure that directly follows the arterial pulse wave, thus neglecting the elastic behaviour of the skin. It will also be important to be able to change the scale of the measurement to provide the information in medical units (e.g. $\mathrm{mmHg}$ ). An artifact reduction algorithm is also needed in order to reduce the fixation requirements for situations in which no contact with the patient is allowed. It is also important to work on a better fixation method, able to be attached directly to different parts of the body for those cases in which the contact is possible. Finally, the processing methods need to be optimized in order to produce real time measurements.

\section{Conclusion}

SMI has been proven as a simple, low cost and accurate technique which enables the measurement of the amplitude profile of the arterial pulse wave and the heart beat frequency. The experimental setup used for this work is compact, cheap and robust, enabling the reconstruction of the pulse shape with an estimated resolution of $400 \mathrm{~nm}(\lambda / 2)$. The reconstruction errors in the method and setup were evaluated using an artificial pulse produced by a piezoelectric stage emulating a real cardiovascular pulse.

It has also been shown that good results can be achieved for long time measurements, which could be useful for real time measurements given that fast reconstruction algorithms are implemented. Also, measurements with different sampling rates were performed showing proper reconstruction for sampling frequencies down to $3.1 \mathrm{kS} / \mathrm{s}$.

\section{Acknowledgement}

The authors would like to thank the Spanish Ministry of Science and Innovation for the funding provided by Plan Nacional I+D+I through project DPI2011-25525. Francisco J. Azcona would also like to thank AGAUR for its funding through pre-doctoral grant 2012FI_BI 00240.

\section{References}

[1] M. Z. Poh, D. J. McDuff and R. W. Picard, Advancements in noncontact, multiparameter physiological measurements using a webcam, IEEE Trans. Biomed. Eng., 58 (2011), pp. 7 - 11.

[2] B. Saugel, A. S. Meidert, A. Hapfelmeier, F. Eyer, R. M. Schmid, W. Huber, Non-invasive continuous arterial pressure measurement based on radial artery tonometry in the intensive 
care unit: a method comparison study using the T-Line TL-200pro device, Brit. J. Anaesth., 111 (2012), pp. 185 - 190.

[3] A. Lang, A. Carass, M. Hauser, E. S. Sotirchos, P. A. Calabresi, H. S. Ying, and J. L. Prince, Retinal layer segmentation of macular OCT images using boundary classification, Biomed Opt Express, 4 (2013), pp. 1133 - 1152.

[4] J. Hast, R. Myllylä, H. Sorvoja, J. Miettinen, Arterial press shape measurement using selfmixing effect in a diode laser, Quantum Electronics, 32 (2002), pp. 975 - 980.

[5] M. F. O'Rourke, A. Pauca and X. Jiang, Pulse wave analysis, Br. J. Clin. Pharmacol., 51 (2001), pp. $507-522$.

[6] M. Saito, M. Matsukawa, T. Asada and Y. Watanabe, Noninvasive assessment of arterial stiffness by pulse wave analysis, IEEE Trans. Ultrason. Ferroelectr., 59 (2012), pp. 2411 - 2419.

[7] S. R. Alty, N. Angarita-Jaimes, S. C. Millasseau and P. J. Chowienczyk, Predicting arterial stiffness from the digital volume pulse waveform, IEEE Trans. Biomed. Eng., 54 (2005), pp. $2268-2275$.

[8] M. Brand, A. J. Woodwiss, F. Michael, H. L. Booysen, M. G. Veller, G. R. Norton, A mismatch between aortic pulse pressure and pulse wave velocity predicts advanced peripheral arterial disease, Eur. J. Vasc. Endovasc., 46 (2013), pp. 338-346.

[9] J. Allen, Photoplethysmography and its application in clinical physiological measurement,Physiol. Meas., 28 (2007), pp. R1-R39.

[10] G. Cennini, J. Arguel, K. Aksit and A. van Leest, Heart rate monitoring via remote photoplethysmography with motion artifacts reduction, Opt. Exp. 18 (2010), pp. 4867-4875.

[11] J. Zheng, S. Hu, V. Azorin-Peris, A. Echiadis, V. Choliaras and R. Summers, Remote simultaneous dual wavelength imaging photoplethysmography: a further step towards 3-D mapping of skin blood microcirculation, in Proc of SPIE 6850 (2008), pp. 6850S-1-6850S-8.

[12] Y. Li, P. Segers, J. Dirckx and R. Baets, On-chip laser Doppler vibrometer for arterial pulse wave velocity measurement, Opt. Exp., 4 (2013), pp. 1229-1235.

[13] J. Hast, R. Myllyl, Sorvoja H., Miettinen J., Self-mixing interferometry in noninvasive pulse wave velocity measurement, Molecular and Quantum Acoustics, 22 (2001), pp. 95 - 106.

[14] K. Meigas, H. Hinrikus, R. Kattai and J. Lass, Self-mixing in a laser diode as a method for cardiovascular diagnosis, J. Biomed. Opt., 8 (2013), pp. 152-160 (2003).

[15] S. Donati and M. Norgia, Self-mixing interferometry for biomedical signals sensing, IEEE J. Sel. Top. Quant., 20 (2014), pp. 6900108 1-8. 
[16] R. Lang and K. Kobayashi, External optical feedback effects on semiconductor laser injection properties, IEEE J. Quantum Electron, 15 (1980), pp. 347 - 355.

[17] W. M. Wang, K. T. V. Grattan, A. W. Palmer and W. J. O. Boyle, Self-mixing interference inside a single mode diode laser for optical sensing applications, J. Lightw. Technol., 12 (1994), pp. 1577 - 1587.

[18] M.Wang, Fourier transform method for self-mixing interference signal analysis, Opt. Laser Technol., 33 (2001), pp. 409 - 416.

[19] A. Acket, D. Leenstra, A. J. Den Boef and B. H. Verbeek, The influence of feedback intensity on longitudinal mode properties and optical noise in index guided semiconductor lasers, IEEE Quantum. Electron., 20 (1984), pp. 1163 - 1169.

[20] U. Zabit, F. Bony, T. Bosch and A. D. Rakic, A self-mixing displacement sensor with fringe loss compensation for harmonics, IEEE Photon. Technol. Lett., 22 (2010), pp. 410 - 412.

[21] S. Donati, Responsivity and noise of self-mixing photodetection schemes, IEEE J. Quantum. Electron., 47 (2011), pp. 1428 - 1433.

[22] R. Atashkhooei, S. Royo, F. J. Azcona and U. Zabit, Analysis and control of speckle effects in self-mixing interferometry, in IEEE Sensors 2011 (2011), pp. 1390 - 1393.

[23] J. Allen and A. Murray, Effects of filtering multi-site photoplethysmography pulse waveform characteristics, in Computers in Cardiology IEEE 2004 (2004), pp. 485 - 488.

[24] C. Bes, G. Plantier and T. Bosch, Displacement measurements using a self-mixing laser diode under moderate feedback, IEEE Trans. Instrum. Meas., 55 (2006), pp. 1101 - 1105.

[25] F. J. Azcona, R. Atashkhooei, S. Royo, J. Mendez Astudillo and A. Jha, A nanometric displacement measurement system using differential optical feedback interferometry, IEEE Photon. Lett.,25 (2013), pp. 2074 - 2077.

[26] S. Donati, G. Martini and T. Tambosso, Speckle pattern errors in self-mixing interferometry, IEEE J. Quantum. Electron., 49 (2001), pp. 798 - 806.

[27] M. Noria, S. Donati and D. d'Alessandro, Interferometric measurements of displacement on a diffusing target by speckle tracking technique, IEEE J. Quantum Electron., 37 (2011), pp. $800-806$.

[28] R. Atashkhooei, S. Royo and F. J. Azcona, Dealing with speckle effects in self-mixing interferometry measurements, IEEE Sensors J., 13 (2013), pp. 1641 - 1647. 